\title{
MicroRNA-339-5p inhibits cell proliferation of acute myeloid leukaemia by directly targeting SOX4
}

\author{
XUEMING SUN ${ }^{1 *}$, HUAQIANG LIU ${ }^{1 *}$, TINGTING LI ${ }^{1}$ and LAIYING QIN ${ }^{2}$ \\ ${ }^{1}$ Department of Hematology, Yidu Central Hospital of Weifang, Weifang, Shandong 262550; \\ ${ }^{2}$ Department of Clinical Laboratory, Jinan Hospital for Infectious Diseases, Jinan, Shandong 250021, P.R. China
}

Received February 15, 2018; Accepted July 25, 2018

DOI: $10.3892 / \mathrm{mmr} .2018 .9552$

\begin{abstract}
In recent decades, microRNAs (miRNAs) have been considered novel gene regulators. Dysregulated miRNAs serve crucial roles in the formation and progression of acute myeloid leukaemia (AML). Therefore, the roles of differentially expressed miRNAs in AML require extensive investigation to obtain insight into the treatment of patients with AML. The present study demonstrated significant miR-339-5p downregulation in AML samples and cell lines. miR-339-5p overexpression attenuated AML cell proliferation by inducing cell cycle arrest and promoting cell apoptosis. Additionally, sex-determining region Y-related high-mobility group box 4 (SOX4) was identified as a direct target gene of miR-339-5p in AML. Furthermore, SOX4 expression was significantly upregulated in AML samples; this upregulation was inversely correlated with the expression levels of miR-339-5p. Additionally, a series of rescue experiments demonstrated that SOX4 resumption reversed the effects of miR-339-5p overexpression on cell proliferation, cycle status and apoptosis of AML. In conclusion, miR-339-5p may serve its antiproliferative role in AML by directly targeting SOX4, which suggests that miR-339-5p may be considered an effective novel therapeutic target for treating patients with such an aggressive haematological malignancy.
\end{abstract}

\section{Introduction}

Acute myeloid leukaemia (AML) is a type of haematological malignancy caused by the malignant transformation of bone-marrow-derived, self-renewing stem cells or myeloid

Correspondence to: Professor Laiying Qin, Department of Clinical Laboratory, Jinan Hospital for Infectious Diseases, 22029 Jingshi Road, Jinan, Shandong 250021, P.R. China

E-mail: jinan_qly@126.com

*Contributed equally

Key words: acute myeloid leukaemia, microRNA-339-5p, proliferation, cell cycle, apoptosis, sex-determining region Y-related high-mobility group box 4 progenitors (1). AML is characterised by cells with unlimited proliferation ability, impaired apoptosis and the accumulation of differentiation-arrested myeloid progenitor cells $(2,3)$. AML is the most common type of leukaemia arising in infancy and childhood, and accounts for $15-20 \%$ cases of acute leukaemia in children (4). At present, chemotherapy, targeted therapy and hematopoietic stem cell transplantation serve as the primary therapeutic methods for patients with AML (5). Notable advancements have been achieved in the diagnosis and therapy of AML; however, the long-term survival of patients with AML remains unsatisfactory (6). Chromosomal aberrations and gene mutations have been associated with the initiation and progression of AML; however, the detailed mechanisms underlying the pathogenesis of AML remain unclear (7). Therefore, the mechanisms underlying AML leukemogenesis and development require further investigation to identify potential therapeutic targets for the treatment of patients with AML and improve the therapeutic outcomes of patients with this particular type of malignancy.

MicroRNAs (miRNAs) are a large family of endogenous, non-coding and short RNA molecules composed of 18-24 nucleotides (8). miRNAs typically regulate gene expression by preferentially interacting with the 3 -untranslated regions (3'-UTRs) of their target genes, thereby inducing mRNA degradation or the inhibition of translation (9). miRNAs serve a principal role in regulating various physiological processes, including cellular growth, differentiation, metabolism, the cell cycle and apoptosis (10-12). Substantial evidence has demonstrated that miRNAs are differently expressed in aapproximately all types of human malignancies, including AML (13-15). In AML, aberrantly expressed miRNAs contribute to the genesis and development of AML by affecting a variety of pathological processes, including cell proliferation, cycle, apoptosis, chemotherapy resistance and autophagy (16-18). Therefore, AML-associated miRNAs require investigation to develop novel diagnostic biomarkers and effective therapeutic targets for the treatment of patients with AML.

miR-339-5p-5p (miR-339-5p) is dysregulated in numerous types of cancer, including non-small cell lung cancer (19), hepatocellular carcinoma (20) and ovarian cancer (21). This dysregulation has been associated with tumorigenesis and tumour development $(19,20,22)$. In addition, miR-339-3p expression is decreased in AML (23); however, the expression, 
roles and detailed mechanism of miR-339-5p in AML require further investigation. In the present study, miR-339-5p was demonstrated to inhibit cell proliferation, induce cell cycle arrest and promote cell apoptosis in AML by directly targeting sex-determining region Y-related high-mobility group box 4 (SOX4). The results of the present study may provide insight into the therapeutic potential of miR-339-5p as a novel effective target for the treatment of patients with AML.

\section{Materials and methods}

Ethics approval and clinical specimens. The present study was approved by the Ethics Committee of the Yidu Central Hospital of Weifang (Weifang, China). Written informed consent was obtained from all patients enrolled in the present study. Clinical specimens were used in accordance with the Declaration of Helsinki. Bone marrow samples were collected from 35 patients with AML (19 males, 16 females; age range, 26-54 years) and 19 healthy controls (12 males, 7 females; age range, 23-47 years) in the Yidu Central Hospital of Weifang from February 2014 to October 2016. Healthy bone marrow tissues were collected form healthy transplantation donors. Patients had not received chemotherapy, targeted therapy or hematopoietic stem cell transplantation prior to bone marrow aspiration. Patiens diagnosed with AMLs that had not been treated with chemotherapy, targeted therapy or hematopoietic stem cell transplantation were enrolled in the present study.

Cell lines. Three AML cell lines (HL-60, THP-1 and Kasumi-1) and a normal bone marrow cell line, HS-5, were obtained from the American Type Culture Collection (Manassas, VA, USA), and were cultured in Dulbecco's modified Eagle's medium (DMEM) containing 10\% heat-inactivated fetal bovine serum (FBS), $100 \mathrm{U} / \mathrm{ml}$ penicillin and $100 \mathrm{mg} / \mathrm{ml}$ streptomycin (all Gibco; Thermo Fisher Scientific, Inc., Waltham, MA, USA). All cell lines were cultured at $37^{\circ} \mathrm{C}$ in a humidified atmosphere with $5 \% \mathrm{CO}_{2}$ and $95 \%$ air.

Cell transfection. Synthetic miR-339-5p mimics and negative control miRNA mimics (miR-NC) were purchased from Shanghai GenePharma Co., Ltd. (Shanghai, China). The miR-339-5p mimics sequence was 5'-UCCCUGUCCUCC AGGAGCUCACG-3' and the miR-NC sequence was 5'-UUC UCCGAACGUGUCACGUTT-3'. The SOX4 overexpression plasmid lacking 3'-UTR, pCMV-SOX4 and an empty plasmid, pCMV, were produced by GeneCopoeia, Inc. (Rockville, MD, USA). Cells were plated into six-well plates at a density of $6 \times 10^{5}$ cells/well one night prior to transfection. Cells were transfected with miR-339-59 mimics (100 pmol), miR-NC (100 pmol), pCMV (4 $\mu \mathrm{g})$ or pCMV-SOX4 (4 $\mu \mathrm{g})$ using Lipofectamine ${ }^{\circledR} 2000$ (Invitrogen; Thermo Fisher Scientific, Inc.) according to the manufacturer's protocols.

Co-transfection of miR-339-59 mimics (100 pmol) and pCMV $(4 \mu \mathrm{g})$ or pCMV-SOX4 $(4 \mu \mathrm{g})$ was additionally conducted using Lipofectamine ${ }^{\circledR} 2000$, according to the manufacturer's protocols. At $8 \mathrm{~h}$ post-transfection, the cell culture medium containing Lipofectamine ${ }^{\circledR} 2000$ was discarded, and fresh DMEM containing 10\% FBS was added into each well. Cells were grown at $37^{\circ} \mathrm{C}$ in a humidified atmosphere containing $5 \% \mathrm{CO}_{2}$ and $95 \%$ air. In the present study, HL-60 and THP-1 cells were selected for functional analysis as the two cell lines exhibited relatively lower miR-339-5p expression. Successful transfection was determined by detecting miR-339-5p and SOX4 expression following transfection using reverse transcription-quantitative polymerase chain reaction (RT-qPCR) and western blot analysis, respectively. RT-qPCR and western blot analysis were performed 48 and $72 \mathrm{~h}$ after transfection, respectively. Following $24 \mathrm{~h}$ transfection, a Cell Counting kit-8 (CCK-8) assay was conducted. Cell cycle and apoptosis assays were conducted $48 \mathrm{~h}$ post-transfection.

RNA isolation and RT-qPCR. Ficoll-Paque Plus (GE Healthcare, Chicago, IL, USA) was utilised to isolate mononuclear cells from the bone marrow samples. Total RNA was extracted from the cultured cell lines and mononuclear cells using TRIzol ${ }^{\circledR}$ reagent (Invitrogen; Thermo Fisher Scientific, Inc.) according to the manufacturer's protocol. Subsequently, the quality and concentration of total RNA was evaluated using a NanoDrop ND-1000 spectrophotometer (NanoDrop Technologies; Thermo Fisher Scientific, Inc.). For the determination of miR-339-5p expression levels, first-strand complementary DNA (cDNA) was prepared from total RNA using a TaqMan MicroRNA RT kit (Applied Biosystems; Thermo Fisher Scientific, Inc.), according to the manufacturer's protocols. Subsequently, $400 \mathrm{ng}$ cDNA was subjected to qPCR using a TaqMan MicroRNA Assay kit (Applied Biosystems; Thermo Fisher Scientific, Inc.). To quantify the levels of SOX4 mRNA expression, a PrimeScript RT Reagent kit (Takara Biotechnology Co., Ltd., Dalian, China) was used to produce cDNA, according to the manufacturer's protocol. Subsequently, qPCR was conducted using a SYBR Premix Ex Taq kit (Takara Biotechnology Co., Ltd., Dalian, China). The thermocycling conditions of qPCR were as follows: $5 \mathrm{~min}$ at $95^{\circ} \mathrm{C}$, followed by 40 cycles of $95^{\circ} \mathrm{C}$ for $30 \mathrm{sec}$ and $65^{\circ} \mathrm{C}$ for $45 \mathrm{sec}$. The relative expression levels of miR-339-5p and SOX4 mRNA were analysed using the $2^{-\Delta \Delta C q}$ method (24) and normalised to U6 and GAPDH, respectively. RT-qPCR analysis was performed on an Applied Biosystems 7500 Sequence Detection system (Thermo Fisher Scientific, Inc.). The primers were designed as follows: miR-339-5p forward, 5'-ACACTCCAGCTGGGTCCCTGTCCTCCA GGAG-3' and reverse, 5'-TGGTGTCGTGGAGTCG-3'; U6 forward, 5'-GCTTCGGCAGCACATATACTAAAAT-3' and reverse, 5'-CGCTTCACGAATTTGCGTGTCAT-3'; SOX4 forward, 5'-CTTGACATGATTAGCTGGCATGAT T-3' and reverse, 5'-CCTGTGCAATATGCCGTGTAGA-3'; and GAPDH forward, 5'-CGGAGTCAACGGATTTGGTCG TAT-3' and reverse, 5'-AGCCTTCTCCATGGTGGTGAA GAC-3'. Each sample was analyzed in triplicate.

CCK-8 assay. Cell proliferation was determined using a CCK-8 assay. Transfected cells were harvested after $24 \mathrm{~h}$ incubation at $37^{\circ} \mathrm{C}$, and seeded into 96 -well plates at a density of 3,000 cells/well. In the present study, four time points were selected: 0, 24, 48 and $72 \mathrm{~h}$ following incubation. At each time point, $10 \mu$ l CCK-8 reagent (Dojindo Molecular Technologies, Inc., Kumamoto, Japan) was added to each well, respectively; cells were incubated at $37^{\circ} \mathrm{C}$ for another $2 \mathrm{~h}$. Finally, the optical density was measured at a wavelength of $450 \mathrm{~nm}$ 


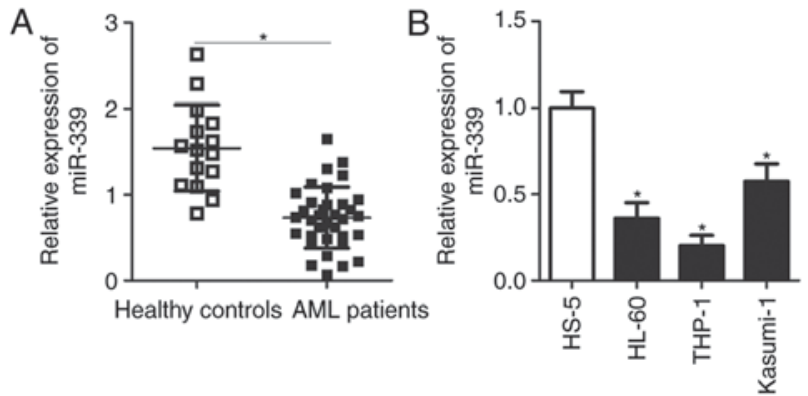

Figure 1. miR-339-5p expression is significantly decreased in AML. (A) Expression levels of miR-339-5p were detected in 35 patients with AML and 19 healthy controls using RT-qPCR. "P $<0.05$ vs. healthy controls. (B) RT-qPCR analysis was conducted to determine the expression levels of miR-339-5p in three AML cell lines (HL-60, THP-1 and Kasumi-1) and a normal bone marrow cell line, HS-5. ${ }^{*} \mathrm{P}<0.05$ vs. HS-5. AML, acute myeloid leukaemia; miR, microRNA; RT-qPCR, reverse transcription-quantitative polymerase chain reaction.

using a Synergy ${ }^{\mathrm{TM}}$ Multi-Mode Microplate Reader (Biotek Instruments, Winooski, VT, USA).

Cell cycle assay. Transfected cells were collected at $48 \mathrm{~h}$ post-transfection, washed with cold PBS and fixed in $70 \%$ ethanol at $4^{\circ} \mathrm{C}$ for $1 \mathrm{~h}$. Following centrifugation at $157 \mathrm{x} \mathrm{g}$ at $4^{\circ} \mathrm{C}$ for $5 \mathrm{~min}$, the supernatant was discarded, and the cells were washed three times with cold PBS. Prior to detection, $50 \mu \mathrm{l}$ RNase $1(100 \mu \mathrm{g} / \mathrm{ml})$ was added to ensure that only the DNA was stained, and this procedure was performed for $10 \mathrm{~min}$ at room temperature. Subsequently, cells were stained at $37^{\circ} \mathrm{C}$ for $30 \mathrm{~min}$ with $25 \mu \mathrm{l}$ propidium iodide solution diluted in $425 \mu \mathrm{l}$ cell staining buffer (both from BioLegend, San Diego, CA, USA), according to the manufacturer's protocol. The cell cycle was detected using a flow cytometer (FACScan; BD Biosciences, Franklin Lakes, NJ, USA), and analysed with CellQuest version 5.1 (BD Biosciences).

Cell apoptosis assay. After $48 \mathrm{~h}$ post-transfection, cells were collected using EDTA-free trypsin (Gibco; Thermo Fisher Scientific, Inc.) and washed three times with cold PBS. Subsequently, an Annexin V-fluorescein isothiocyanate (FITC) Apoptosis Detection kit (BioLegend) was utilized to detect cell apoptosis, according to the manufacturer's protocols. Transfected cells $\left(1.5 \times 10^{6}\right)$ were re-suspended in $100 \mu \mathrm{l}$ binding buffer, followed by incubation with $5 \mu \mathrm{l}$ Annexin V-FITC and $5 \mu \mathrm{l}$ propidium iodide in the dark for $15 \mathrm{~min}$ at room temperature. Finally, cell apoptosis rate was detected using a flow cytometer (FACScan), and analyzed with CellQuest version 5.1 (both BD Biosciences, Franklin Lakes, NJ, USA).

Bioinformatics analysis. TargetScan7.1 (http://www. targetscan.org/) was used to predict the putative targets of miR-339-5p.

Dual-luciferase reporter assay. SOX4 was predicted as a potential target of miR-339-5p using the bioinformatics tools. The fragments of the SOX4 3'-UTR containing wild-type (Wt) or mutant (Mut) binding sites of miR-339-5p were chemically produced by Shanghai GenePharma Co., Ltd., and inserted into pGL3 luciferase reporter vectors (Promega Corporation, Madison, WI, USA) and named as pGL3-SOX4-3'-UTR Wt and pGL3-SOX4-3'-UTR Mut, respectively. Cells were inoculated into $24-w e l l$ plates with a density of $1.0 \times 10^{5}$ cells/well one day prior to transfection. miR-339-5p mimics (50 pmol) or miR-NC (50 pmol) were co-transfected with pGL3-SOX4-3'-UTR Wt $(0.2 \mu \mathrm{g})$ or pGL3-SOX4-3'-UTR Mut $(0.2 \mu \mathrm{g})$ into cells using Lipofectamine ${ }^{\circledR} 2000$, according to the manufacturer's protocol. After 48-h transfection, Renilla and firefly luciferase activities were evaluated using a Dual-Luciferase Reporter Assay kit (Promega Corporation). Renilla luciferase activity was employed for normalisation of luciferase activity.

Western blot analysis. Total protein was extracted from cultured cells or bone marrow samples using radioimmunoprecipitation assay lysis buffer (Beyotime Institute of Biotechnology, Shanghai, China). A Bicinchoninic Acid assay kit (Beyotime Institute of Biotechnology) was used to evaluate protein concentration. Equal amounts of proteins $(30 \mu \mathrm{g})$ were separated via $10 \%$ SDS-PAGE and transferred to polyvinylidene fluoride membranes (EMD Millipore, Billerica, MA, USA). Following blocking with 5\% non-fat dry milk at room temperature for $1 \mathrm{~h}$, the membranes were incubated with primary antibodies overnight at $4^{\circ} \mathrm{C}$ and subsequently washed three times with Tris-buffered saline containing 0.1\% Tween-20 (TBST). Subsequently, the membranes were incubated with a horseradish-peroxidase-conjugated secondary antibody (1:5,000; cat. no. ab6789; Abcam, Cambridge, UK) for $2 \mathrm{~h}$ at room temperature and washed three times with TBST. Target protein signals were visualised using Amersham Enhanced Chemilumiscence Western Blotting Detection Reagent (GE Healthcare) according to the manufacturer's protocols. The primary antibodies used in the present study included mouse anti-human monoclonal SOX4 (1:500; cat. no. ab70598) and mouse anti-human monoclonal GAPDH antibody (1:500; cat. no. ab8245; both Abcam); GADPH was used as the internal reference. Protein expression was quantified using Quantity One software version 4.62 (Bio-Rad Laboratories, Inc., Hercules, CA, USA).

Statistical analysis. Data are expressed as the mean \pm standard deviation and analyzed with SPSS 16.0 software (SPSS Inc., Chicago, IL, USA). Each experiment was repeated at least three times. Differences between groups were determined using Student's t-test or one-way analysis of variance for multiple comparisons followed by Tukey's post-hoc test. Spearman's correlation analysis was performed to assess the correlation between miR-339-5p and SOX4 mRNA expression levels in AML. $\mathrm{P}<0.05$ was considered to indicate a statistically significant difference.

\section{Results}

miR-339-5p is downregulated in AML. To assess the cellular functions of miR-339-5p in AML, the expression levels of miR-339-5p in the bone marrow of 35 patients with AML and 19 healthy controls were analysed. RT-qPCR analysis demonstrated that miR-339-5p was significantly downregulated in patients with AML compared with in healthy controls (Fig. 1A; $\mathrm{P}<0.05)$. The expression levels of miR-339-5p were detected in 
A

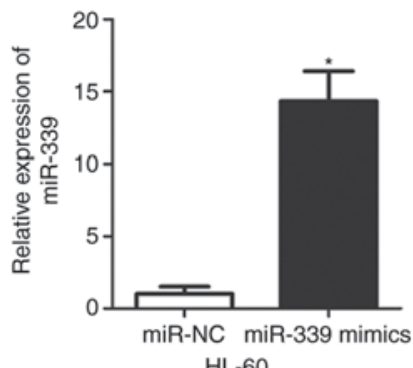

$\mathrm{HL}-60$

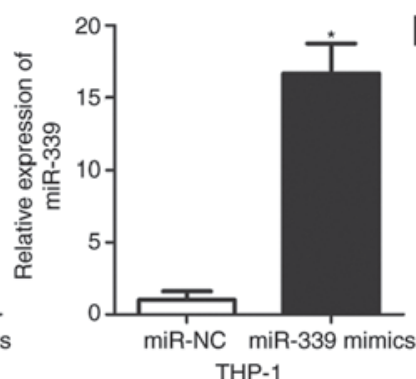

THP-1
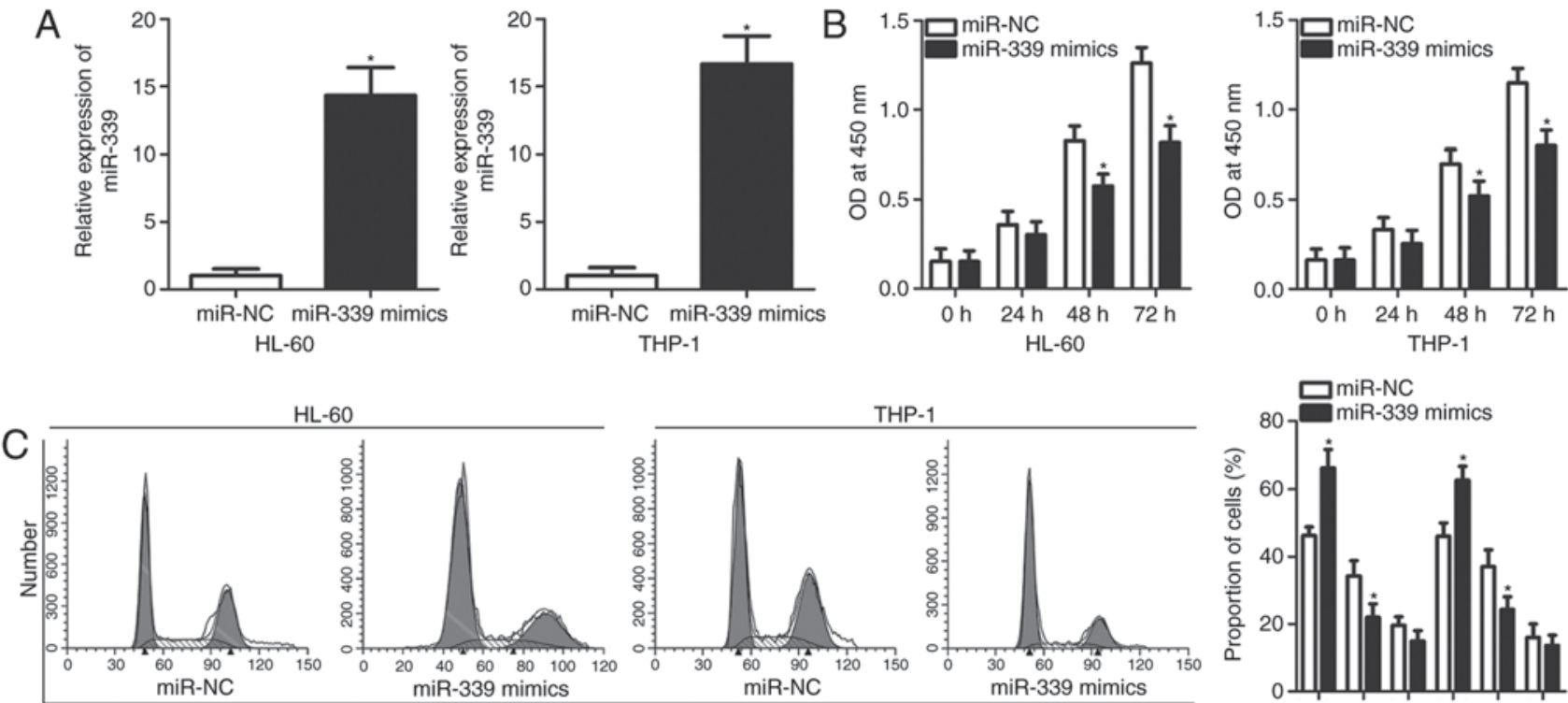

$\mathrm{HP}-1$

Channels (FL2-A)

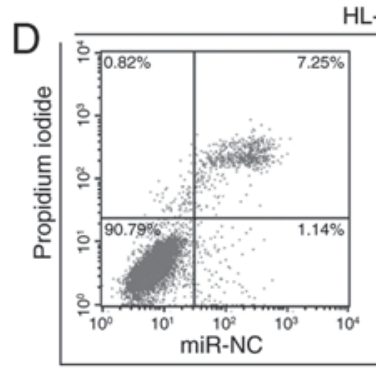

HL-60
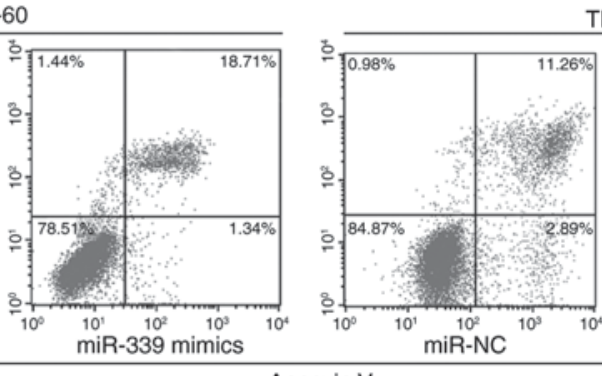

THP-
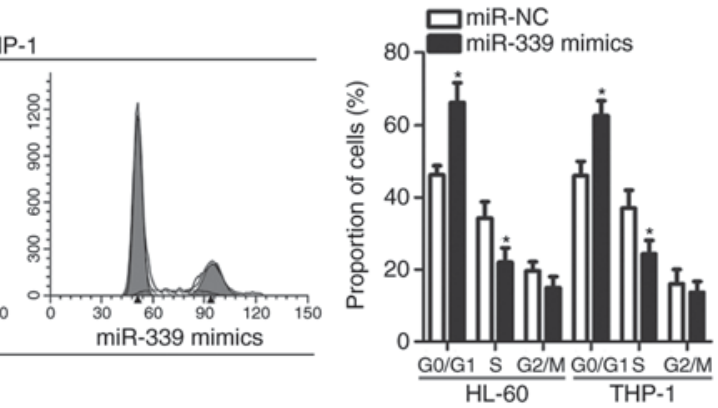

Annexin V
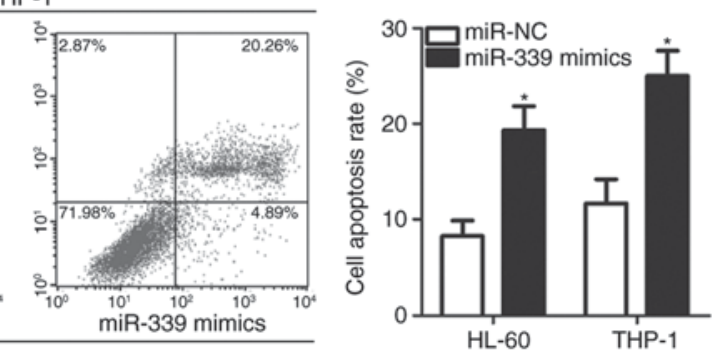

Figure 2. Upregulation of miR-339-5p inhibits cell proliferation of acute myeloid leukaemia. (A) HL-60 and THP-1 cells were transfected with miR-339-5p mimics or miR-NC. At $48 \mathrm{~h}$ post-transfection, the transfection efficiency was evaluated via reverse transcription-quantitative polymerase chain reaction. ${ }^{*} \mathrm{P}<0.05$ vs. miR-NC. (B) Proliferative abilities of HL-60 and THP-1 cells transfected with miR-339-5p mimics or miR-NC were determined via a Cell Counting kit- 8 assay. "P<0.05 vs. miR-NC. (C) Cell cycle status of HL-60 and THP-1 cells transfected with miR-339-5p mimics or miR-NC as analyzed by a cell cycle assay. ${ }^{*} \mathrm{P}<0.05$ vs. miR-NC. (D) Cell apoptosis assay was used to assess the effect of miR-339-5p overexpression on the rate of apoptosis of HL-60 and THP-1 cells transfected with miR-339-5p mimics or miR-NC. ${ }^{*} \mathrm{P}<0.05$ vs. miR-NC. miR, microRNA; NC, negative control; OD, optical density.

three AML cell lines (HL-60, THP-1 and Kasumi-1) and in a normal bone marrow cell line HS-5. The expression levels of miR-339-5p in the three AML cell lines were significantly lower compared with HS-5 cells (Fig. 1B; P<0.05). These results suggested that miR-339-5p may serve crucial roles in the development of AML.

miR-339-5p overexpression inhibits the proliferation of $A M L$ cells. As it was determined that miR-339-5p is expressed at low levels in AML in the present study, whether miR-339-5p serves a tumour-suppressing role in AML progression was investigated. To confirm this hypothesis, miR-339-5p mimics or miR-NC were transfected into HL-60 and THP-1 cells, and the results of RT-qPCR demonstrated that the miR-339-5p expression levels were significantly upregulated in HL-60 and THP-1 cells transfected with miR-339-5p mimics compared with the control (Fig. 2A; $\mathrm{P}<0.05$ ). The effects of miR-339-5p overexpression on AML cell proliferation was determined via a CCK-8 assay. As presented in Fig. 2B, ectopic miR-339-5p expression significantly inhibited the proliferation of HL-60 and THP-1 cells relative to those of the miR-NC groups at 48 and $72 \mathrm{~h}(\mathrm{P}<0.05)$. Alterations in proliferation were primarily associated with cell cycle progression, suggesting that cell cycle arrest may inhibit the cell cycle. Cell cycle analysis demonstrated that HL-60 and THP-1 cells transfected with miR-339-5p mimics exhibited a significantly higher percentage of cells in the $G_{0} / G_{1}$ phase and lower percentage of cells in the $\mathrm{S}$ phase compared with cells transfected with miR-NC (Fig. $2 \mathrm{C}$; $\mathrm{P}<0.05$ ). A cell apoptosis assay was conducted to examine the biological functions of miR-339-5p in AML cell apoptosis. The results demonstrated that restoration of miR-339-5p expression increased the percentage of apoptotic rate in HL-60 and THP-1 cells compared with cells transfected with miR-NC (Fig. 2D; P<0.05). These results suggested that miR-339-5p may inhibit AML cell proliferation by inducing cell apoptosis and cell cycle arrest.

SOX4 serves as a direct target gene of miR-339-5p in AML. It was identified that miRNAs serve their roles by directly interacting with the 3'-UTR of target genes and induce mRNA degradation or the inhibition of translation (9). Therefore, bioinformatics analysis was performed to predict the potential targets of miR-339-5p in the present study. SOX4, a key 

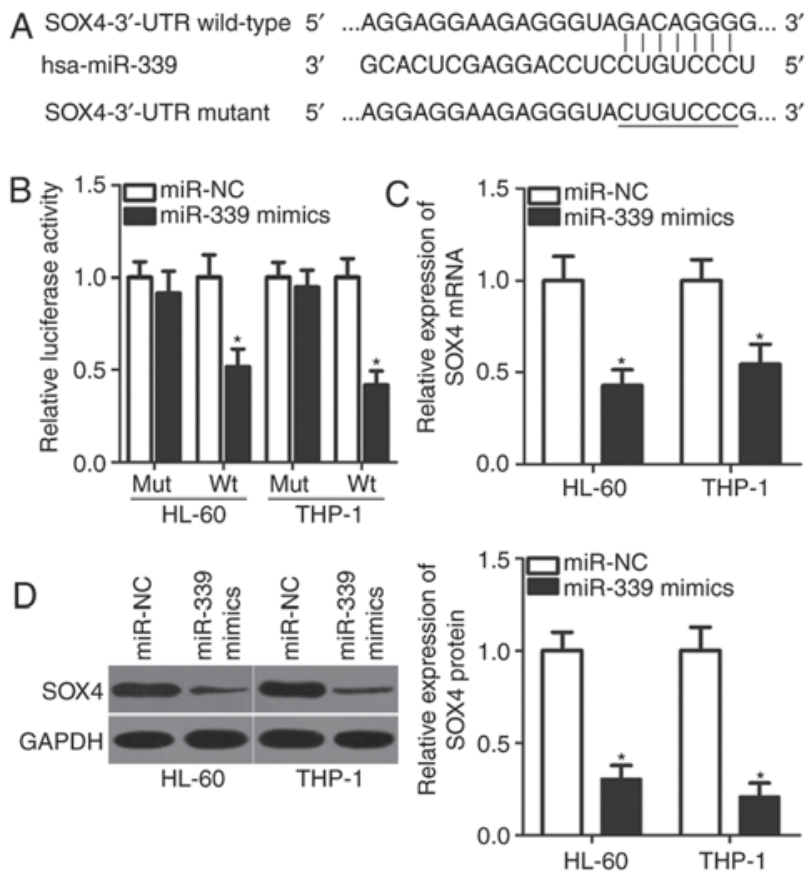

Figure 3. SOX4 is a direct target of miR-339-5p in acute myeloid leukaemia cells. (A) Predicted Wt complementary sequence interaction between miR-339-5p and the 3 '-UTR of SOX4. Mut binding sequences are also presented. (B) HL-60 and THP-1 cells were transfected with the Wt or Mut SOX4 3'-UTR-containing luciferase reporter plasmid combined with miR-339-5p mimics or miR-NC. After $48 \mathrm{~h}$ of transfection, the relative luciferase activity was detected using Dual-Luciferase Reporter Assay kit. ${ }^{*} \mathrm{P}<0.05$ vs. miR-NC. (C) Relative mRNA and (D) protein expression levels of SOX4 in HL-60 and THP-1 cells transfected with miR-339-5p mimics or miR-NC were analysed using reverse transcription-quantitative polymerase chain reaction and western blot analysis, respectively. "P $<0.05$ vs. miR-NC. miR, microRNA; Mut, mutant; NC, negative control; SOX4, sex-determining region Y-related high-mobility group box 4; UTR, untranslated region; Wt, wild type.

component of AML oncogenesis and progression (25-28), was predicted as a putative target of miR-339-5p (Fig. 3A) and was selected for subsequent analysis. A dual-luciferase reporter assay was used to verify the direct interaction between miR-339-5p and the 3'-UTR of SOX4. The data demonstrated that miR-339-5p significantly decreased the luciferase activities of miR-399-50 mimics-transfected HL-60 and THP-1 cells containing the Wt 3 '-UTR of SOX4 compared with in the corresponding control $(\mathrm{P}<0.05)$, whereas the luciferase activity of the plasmid containing the Mut 3'-UTR of SOX4 remained notably unaltered (Fig. 3B). To evaluate the regulatory effects of miR-339-5p on the endogenous expression levels of SOX4, RT-qPCR and western blot analysis were performed to measure SOX4 expression in HL-60 and THP-1 cells transfected with miR-339-5p mimics or miR-NC. As presented in Fig. $3 \mathrm{C}$ and $\mathrm{D}$, the mRNA $(\mathrm{P}<0.05)$ and protein $(\mathrm{P}<0.05)$ expression levels of SOX4 in HL-60 and THP-1 cells transfected with miR-339-5p mimics were significantly lower compared with in cells transfected with miR-NC. Therefore, SOX4 may serve as a direct target of miR-339-5p in AML.

miR-339-5p is negatively correlated with SOX4 expression in $A M L$. To clarify the association between miR-339-5p and SOX4 in AML, the present study investigated SOX4 expression in bone marrow samples of 35 patients with AML and
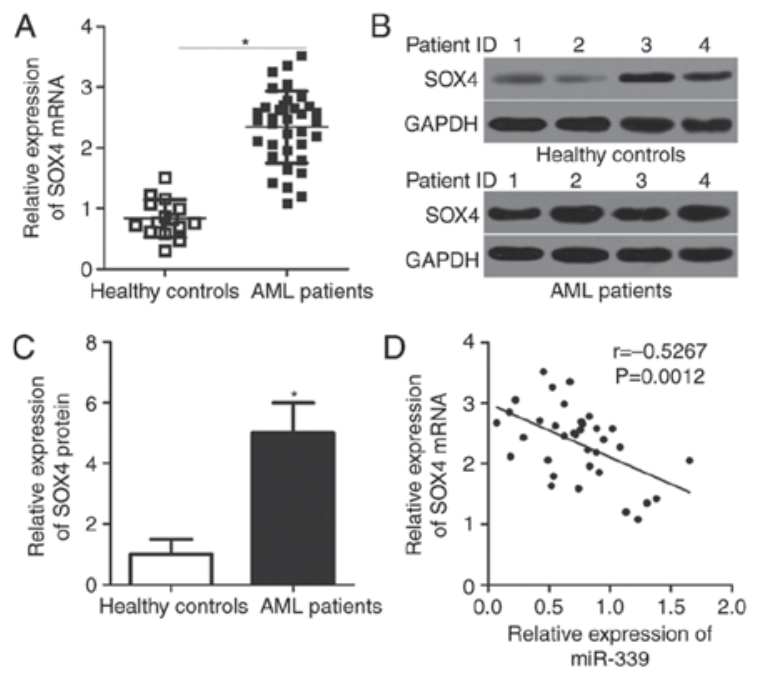

Figure 4. miR-339-5p expression is negatively correlated with SOX4 expression in AML. SOX 4 mRNA and protein expression levels of patients with AML and healthy controls were examined by (A) reverse transcription-quantitative polymerase chain reaction and (B) western blotting, with (C) subsequent analysis, respectively. ${ }^{*} \mathrm{P}<0.05$ vs. healthy controls. (D) Correlation between miR-339-5p and SOX4 mRNA expression in AML samples was determined using Spearman's correlation analysis. $r=-0.5267, P=0.0012$. AML, acute myeloid leukaemia; miR, microRNA; SOX4, sex-determining region Y-related high-mobility group box 4.

19 healthy controls. RT-qPCR analysis demonstrated that SOX4 mRNA was significantly overexpressed in patients with AML compared with in healthy controls (Fig. 4A; $\mathrm{P}<0.05$ ). The protein expression levels of SOX4 in patients with AML were significantly higher compared with the healthy controls (Fig. 4B and C; $\mathrm{P}<0.05$ ). Spearman's correlation analysis suggested that miR-339-5p was inversely correlated with SOX4 mRNA expression in AML samples (Fig. 4D; r=-0.5267, $\mathrm{P}=0.0012)$. These results further suggested that SOX4 is a direct target of miR-339-5p in AML.

SOX4 overexpression partially rescues the inhibitory effects of miR-339-5p on AML cells. Following the determination of SOX4 as a target of miR-339-5p, the present study investigated whether SOX4 mediates the tumour-suppressing roles of miR-339-5p in AML. HL-60 and THP-1 cells were transfected with pCMV or pCMV-SOX4. The results demonstrated that SOX4 expression was significantly upregulated in pCMV-SOX4-transfected HL-60 and THP-1 cells compared with cells transfected with pCMV (Fig. 5A; $\mathrm{P}<0.05$ ). Subsequently, HL-60 and THP-1 cells were co-transfected with miR-339-5p mimics and pCMV or pCMV-SOX4 lacking 3'-UTR. After 72-h transfection, western blot analysis suggested that the downregulation of SOX4 expression in HL-60 and THP-1 cells induced by miR-339-5p overexpression was recovered following co-transfection with pCMV-SOX4 (Fig. 5B; $\mathrm{P}<0.05$ ). In functional assays, the restoration of SOX4 expression significantly reversed the effects of exogenous miR-339-5p on the proliferation at 48 and $72 \mathrm{~h}$ (Fig. 5C; $\mathrm{P}<0.05)$, the cell cycle (Fig. 5D; $\mathrm{P}<0.05$ ) and apoptosis (Fig. 5E; P<0.05) of HL-60 and THP-1 cells. Overall, these results suggested that the tumour-suppressing effects of miR-339-5p on AML cells were associated with downregulated SOX4 expression. 
A

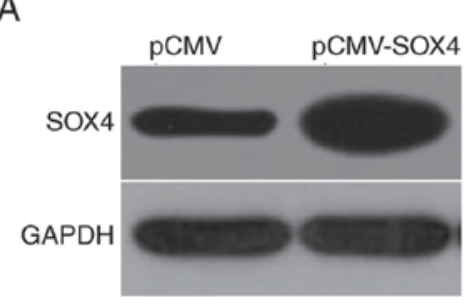

HL-60

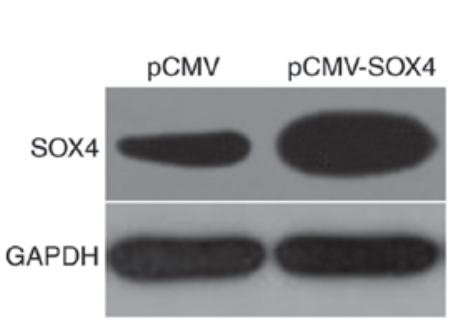

THP-1
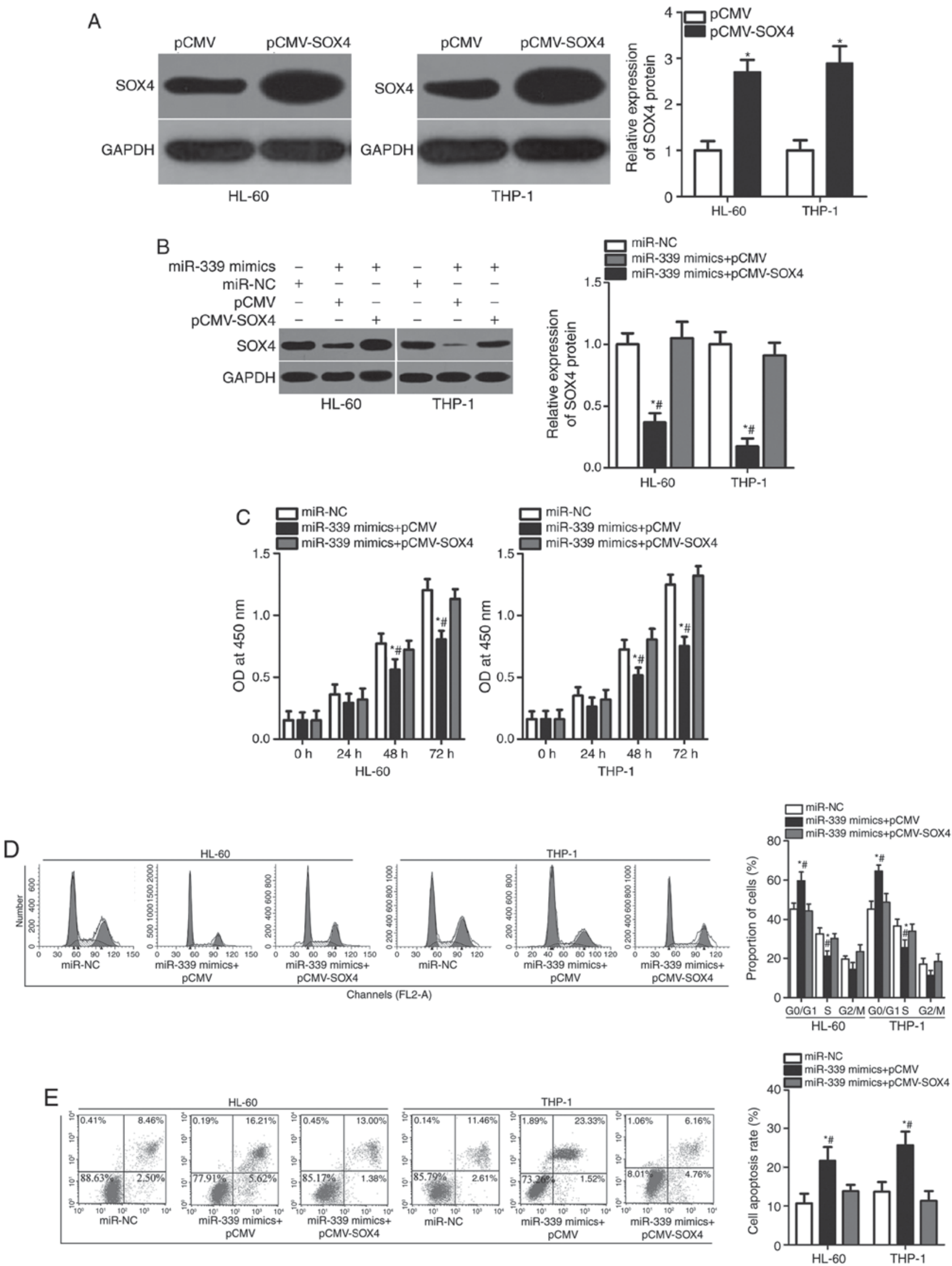

E

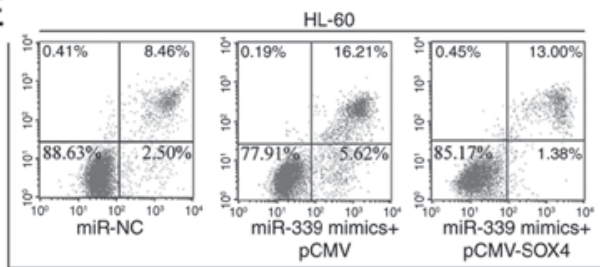

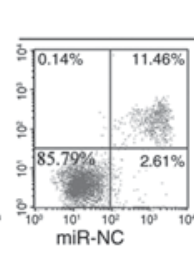

THP-1

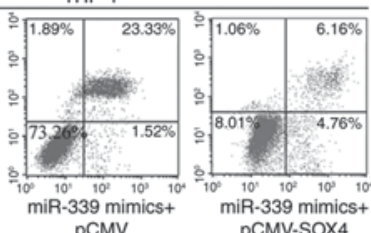




\section{Discussion}

In recent decades, miRNAs have been considered novel gene regulators, whereas deregulated miRNAs serve key roles in the initiation and progression of AML (29-31). Therefore, the roles of differently expressed miRNAs in AML require extensive investigation to gain insight into potential treatments for patients with AML. miR-339-5p has been studied in acute lymphoblastic leukaemia (32); however, its expression profile in AML remains unknown. In the present study, miR-339-5p expression was significantly decreased in AML samples and cell lines. In vitro analyses demonstrated that resumption of miR-339-5p expression reduced cell proliferation, induced cell cycle arrest and promoted apoptosis in AML in the present study. SOX4 was determined to be a direct target of miR-339-5p in AML and was overexpressed in AML samples. This overexpression was inversely correlated with miR-339-5p expression. In addition, the present study conducted a series of rescue experiments, which revealed that the restoration of SOX4 expression inhibited the effects of miR-339-5p overexpression on cell proliferation, the cell cycle and apoptosis of AML. These results demonstrated that miR-339-5p may serve tumour-suppressing roles in AML progression by directly targeting SOX4, suggesting that miR-339-5p may be considered as a therapeutic target for the treatment of patients with AML.

The dysregulation of miR-339-5p has been identified in a variety of human cancer (19-21). FomiR-339-5p was observed to be downregulated in non-small cell lung cancer tissues and cell lines (19). Reduced miR-339-5p expression has been associated with tumour-node-metastasis staging and lymph node metastasis (19). The expression levels of miR-339-5p in hepatocellular carcinoma were lower compared with tumour tissues and cell lines. Compared with in patients with hepatocellular carcinoma and high miR-339-5p expression levels, those with lower miR-339-5p expression levels present poorer prognosis (20). miR-339-5p expression levels have been proposed as an independent prognostic factor for patients with hepatocellular carcinoma (20). Low expression levels of miR-339-5p were also reported in ovarian (21), gastric (33) and breast cancer (34). In the present study, it was demonstrated that miR-339-5p expression was decreased in AML samples and cell lines. These results suggested that miR-339 is frequently downregulated in human malignancies and may be associated with the pathogenesis of these specific types of tumour.

It was demonstrated that differentially expressed miRNAs are closely correlated with the carcinogenesis and progression of numerous human cancer types $(19,20,35,36)$. For instance, upregulation of miR-339-5p inhibited the cell proliferation, migration and invasion of lung cancer $(19,35)$. Zhou et al (36) observed that miR-339-5p overexpression decreased the growth, colony formation and metastasis of colorectal cancer cell in vitro and suppressed tumour growth in vivo. Wang et al (20) observed that the ectopic expression of miR-339-5p restricted the invasive ability of hepatocellular carcinoma cells. Shan et al (21) demonstrated that the resumption of miR-339-5p expression suppressed cell motility in ovarian cancer. Shen et al (33) identified that miR-339-5p restoration reduced the cell proliferation, migration and invasion of gastric cancer in vitro and tumorigenicity in vivo. Wu et al (34) demonstrated that miR-339-5p overexpression reduced the cell metastatic ability of breast cancer. In the present study, it was observed that miR-339-5p inhibited cell proliferation, induced cell cycle arrest and promoted apoptosis in AML. These results suggested that miR-339-5p may be a potential therapeutic target for the treatment of patients with these specific types of tumour.

Previous studies have validated a number of miR-339-5p targets, including S-phase kinase-associated protein 2 in lung cancer (35), phosphatase of regenerating liver-1 in colorectal cancer (36), nucleus accumbens associated 1 (21) and B-cell lymphoma 6 in ovarian cancer (21) and NOVA alternative splicing regulator 1 in gastric cancer (33). SOX4, a member of the SOX family (37), was observed to be a direct and functional target of miR-339-5p in AML in the present study. Increasing evidence suggests that SOX4 is aberrantly and highly expressed in numerous types of human cancers, including gastric cancer (38), hepatocellular carcinoma (39), colorectal cancer (40), osteosarcoma (41) and cervical cancer (42). SOX4 serves oncogenic roles in the onset and development of tumours by affecting cell proliferation, apoptosis, the cell cycle, migration and metastasis (43-45). SOX4 expression is additionally upregulated in the bone marrow of patients with AML as determined in the preset study. Compared with in patients with low levels of SOX4 expression, those with high expression levels exhibited lower rates of remission and shorter overall survival (25). Functionally, SOX4 performs important roles in the formation and progression of $\mathrm{AML}$, regulating cell self-renewal, differentiation and proliferation (26-28). In view of these important roles, in AML, the miR-339-5p/SOX4 signalling pathway may represent a potential therapeutic target for treating patients with AML.

To summarize, miR-339-5p was downregulated in AML samples and cell lines. miR-339-5p overexpression inhibited cell proliferation, induced cell cycle arrest and increased apoptosis of AML by directly targeting SOX4. The association between miR-339-5p and the prognosis of patients with AML was not investigated in the present study. In addition, the association between miR-339-5p and the clinicopathological features of patients with AML was not examined. These limitations of the present study may be resolved in future investigations; however, understanding the mechanism underlying the tumour-suppressing roles of miR-339-5p in AML may provide novel insight into the progression of AML. miR-339-5p may be considered a potential therapeutic target for the management of patients with AML.

\section{Acknowledgements}

Not applicable.

\section{Funding}

No funding was received.

\section{Availability of data and materials}

The datasets used and/or analyzed during the present study are available from the corresponding author on reasonable request. 


\section{Authors' contributions}

LQ made substantial contributions to the design of the present study. XS, HL and TL performed the functional experiments. All authors read and approved the final manuscript.

\section{Ethics approval and consent to participate}

The present study was approved by the Research Ethics Committee of Yidu Central Hospital of Weifang, and was performed in accordance with the Declaration of Helsinki and the guidelines of the Ethics Committee of Yidu Central Hospital of Weifang. Written informed consent was obtained from all patients for the use of their clinical tissues.

\section{Patient consent for publication}

Not applicable.

\section{Competing interests}

The authors declare that they have no competing interests.

\section{References}

1. Ziai JM and Siddon AJ; Education Committee of the Academy of Clinical Laboratory Physicians and Scientists: Pathology consultation on gene mutations in acute myeloid leukemia. Am J Clin Pathol 144: 539-554, 2015.

2. Estey EH: Acute myeloid leukemia: 2013 update on risk-stratification and management. Am J Hematol 88: 318-327, 2013.

3. Estey E and Döhner H: Acute myeloid leukaemia. Lancet 368: 1894-1907, 2006.

4. Medinger M, Lengerke C and Passweg J: Novel prognostic and therapeutic mutations in acute myeloid leukemia. Cancer Genomics Proteomics 13: 317-329, 2016.

5. Chiu CF, Weng JR, Jadhav A, Wu CY, Sargeant AM and Bai LY: T315 decreases acute myeloid leukemia cell viability through a combination of apoptosis induction and autophagic cell death. Int J Mol Sci 17: pii: E1337, 2016.

6. Liu X, Liao W, Peng H, Luo X, Luo Z, Jiang H and Xu L: miR-181a promotes G1/S transition and cell proliferation in pediatric acute myeloid leukemia by targeting ATM. J Cancer Res Clin Oncol 142: 77-87, 2016.

7. Estey EH: Acute myeloid leukemia: 2014 update on risk-stratification and management. Am J Hematol 89: 1063-1081, 2014.

8. Alemdehy MF and Erkeland SJ: MicroRNAs: Key players of normal and malignant myelopoiesis. Curr Opin Hematol 19: 261-267, 2012

9. Ameres SL and Zamore PD: Diversifying microRNA sequence and function. Nat Rev Mol Cell Biol 14: 475-488, 2013.

10. Carthew RW and Sontheimer EJ: Origins and mechanisms of miRNAs and siRNAs. Cell 136: 642-655, 2009.

11. Bartel DP: MicroRNAs: Genomics, biogenesis, mechanism, and function. Cell 116: 281-297, 2004.

12. Huntzinger $\mathrm{E}$ and Izaurralde $\mathrm{E}$ : Gene silencing by microRNAs: Contributions of translational repression and mRNA decay. Nat Rev Genet 12: 99-110, 2011.

13. Nagai H, Hasegawa S, Uchida F, Terabe T, Ishibashi Kanno N, Kato K, Yamagata K, Sakai S, Kawashiri S, Sato H, et al: MicroRNA-205-5p suppresses the invasiveness of oral squamous cell carcinoma by inhibiting TIMP-2 expression. Int J Oncol 52: 841-850, 2018.

14. Du B, Wu D, Yang X, Wang T, Shi X, Lv Y, Zhou Z, Liu Q and Zhang W: The expression and significance of microRNA in different stages of colorectal cancer. Medicine (Baltimore) 97: e9635, 2018.

15. Guo Q, Luan J, Li N, Zhang Z, Zhu X, Zhao L, Wei R, Sun L, Shi Y, Yin X, et al: MicroRNA-181 as a prognostic biomarker for survival in acute myeloid leukemia: A meta-analysis. Oncotarget 8: 89130-89141, 2017.
16. Liu L, Ren W and Chen K: MiR-34a promotes apoptosis and inhibits autophagy by targeting HMGB1 in acute myeloid leukemia cells. Cell Physiol Biochem 41: 1981-1992, 2017.

17. Xiao Y, Deng T, Su C and Shang Z: MicroRNA 217 inhibits cell proliferation and enhances chemosensitivity to doxorubicin in acute myeloid leukemia by targeting KRAS. Oncol Lett 13: 4986-4994, 2017.

18. Wallace JA and O'Connell RM: MicroRNAs and acute myeloid leukemia: Therapeutic implications and emerging concepts. Blood 130: 1290-1301, 2017.

19. Li Y, Zhao W, Bao P, Li C, Ma XQ, Li Y and Chen LA: miR-339-5p inhibits cell migration and invasion in vitro and may be associated with the tumor-node-metastasis staging and lymph node metastasis of non-small cell lung cancer. Oncol Lett 8: 719-725, 2014.

20. Wang YL, Chen CM, Wang XM and Wang L: Effects of miR-339-5p on invasion and prognosis of hepatocellular carcinoma. Clin Res Hepatol Gastroenterol 40: 51-56, 2016.

21. Shan W, Li J, Bai Y and Lu X: miR-339-5p inhibits migration and invasion in ovarian cancer cell lines by targeting NACC1 and BCL6. Tumour Biol 37: 5203-5211, 2016.

22. Zhou C, Lu Y and Li X: miR-339-3p inhibits proliferation and metastasis of colorectal cancer. Oncol Lett 10: 2842-2848, 2015.

23. Barrera-Ramirez J, Lavoie JR, Maganti HB, Stanford WL, Ito C, Sabloff M, Brand M, Rosu-Myles M, Le Y and Allan DS: Micro-RNA profiling of exosomes from marrow-derived mesenchymal stromal cells in patients with acute myeloid leukemia: Implications in leukemogenesis. Stem Cell Rev 13: 817-825, 2017.

24. Livak KJ and Schmittgen TD: Analysis of relative gene expression data using real-time quantitative PCR and the 2(-Delta Delta C(T)) method. Methods 25: 402-408, 2001.

25. Lu JW, Hsieh MS, Hou HA, Chen CY, Tien HF and Lin LI: Overexpression of SOX 4 correlates with poor prognosis of acute myeloid leukemia and is leukemogenic in zebrafish. Blood Cancer J 7: e593, 2017.

26. Fung TK, Leung AY and So CW: Sox4you: A new player in C/EBPa leukemia. Cancer Cell 24: 557-559, 2013.

27. Fernando TR, Contreras JR, Zampini M, Rodriguez-Malave NI, Alberti MO, Anguiano J, Tran TM, Palanichamy JK, Gajeton J, Ung NM, et al: The lncRNA CASC15 regulates SOX4 expression in RUNX1-rearranged acute leukemia. Mol Cancer 16: $126,2017$.

28. Zhang H, Alberich-Jorda M, Amabile G, Yang H, Staber PB, Di Ruscio A, Welner RS, Ebralidze A, Zhang J, Levantini E, et al: Sox4 is a key oncogenic target in $\mathrm{C} / \mathrm{EBP} \alpha$ mutant acute myeloid leukemia. Cancer Cell 24: 575-588, 2013.

29. Gabra MM and Salmena L: microRNAs and acute myeloid leukemia chemoresistance: A mechanistic overview. Front Oncol 7: 255, 2017.

30. Wang X, Chen H, Bai J and He A: MicroRNA: An important regulator in acute myeloid leukemia. Cell Biol Int 41: 936-945, 2017.

31. Yeh $\mathrm{CH}$, Moles $\mathrm{R}$ and Nicot $\mathrm{C}$ : Clinical significance of microRNAs in chronic and acute human leukemia. Mol Cancer 15: 37, 2016.

32. Mosakhani N, Missiry ME, Vakkila E, Knuutila S and Vakkila J: Low expression of miR-18a as a characteristic of pediatric acute lymphoblastic leukemia. J Pediatr Hematol Oncol 39: 585-588, 2017.

33. Shen B, Zhang Y, Yu S, Yuan Y, Zhong Y, Lu J and Feng J: MicroRNA-339, an epigenetic modulating target is involved in human gastric carcinogenesis through targeting NOVA1. FEBS Lett 589: 3205-3211, 2015.

34. Wu ZS, Wu Q, Wang CQ, Wang XN, Wang Y, Zhao JJ, Mao SS, Zhang GH, Zhang N and Xu XC: MiR-339-5p inhibits breast cancer cell migration and invasion in vitro and may be a potential biomarker for breast cancer prognosis. BMC Cancer 10: 542, 2010.

35. Ren H, Zhang Y and Zhu H: MiR-339 depresses cell proliferation via directly targeting $\mathrm{S}$-phase kinase-associated protein 2 mRNA in lung cancer. Thorac Cancer 9: 408-414, 2018.

36. Zhou C, Liu G, Wang L, Lu Y, Yuan L, Zheng L, Chen F, Peng F and $\mathrm{Li} \mathrm{X}$ : MiR-339-5p regulates the growth, colony formation and metastasis of colorectal cancer cells by targeting PRL-1. PLoS One 8: e63142, 2013.

37. Shi S, Cao X, Gu M, You B, Shan Y and You Y: Upregulated expression of SOX4 is associated with tumor growth and metastasis in nasopharyngeal carcinoma. Dis Markers 2015: 658141, 2015. 
38. Fang CL, Hseu YC, Lin YF, Hung ST, Tai C, Uen YH and Lin KY: Clinical and prognostic association of transcription factor SOX4 in gastric cancer. PLoS One 7: e52804, 2012.

39. Zheng JH, Jian ZX, Jin HS, Chen SC and Wang GY: Expression of SOX4 gene and early recurrence of hepatocellular carcinoma: Their relationship and the clinical significance. Nan Fang Yi Ke Da Xue Xue Bao 30: 818-819, 2010 (In Chinese).

40. Wang B, Li Y, Tan F and Xiao Z: Increased expression of SOX4 is associated with colorectal cancer progression. Tumour Biol 37 9131-9137, 2016.

41. Bao ZQ, Zhang CC, Xiao YZ, Zhou JS, Tao YS and Chai DM: Over-expression of Sox 4 and $\beta$-catenin is associated with a less favorable prognosis of osteosarcoma. J Huazhong Univ Sci Technolog Med Sci 36: 193-199, 2016.
42. Sun R, Jiang B, Qi H, Zhang X, Yang J, Duan J, Li Y and Li G: SOX4 contributes to the progression of cervical cancer and the resistance to the chemotherapeutic drug through ABCG2. Cell Death Dis 6: e1990, 2015.

43. Aaboe M, Birkenkamp-Demtroder K, Wiuf C, Sørensen FB, Thykjaer T, Sauter G, Jensen KM, Dyrskjøt L and Ørntoft T: SOX4 expression in bladder carcinoma: Clinical aspects and in vitro functional characterization. Cancer Res 66: 3434-3442, 2006.

44. Zou J and Xu Y: MicroRNA-140 inhibits cell proliferation in gastric cancer cell line HGC-27 by suppressing SOX4. Med Sci Monit 22: 2243-2252, 2016.

45. Cheng Q, Wu J, Zhang Y, Liu X, Xu N, Zuo F and Xu J: SOX4 promotes melanoma cell migration and invasion though the activation of the NF- $\kappa \mathrm{B}$ signaling pathway. Int J Mol Med 40: 447-453, 2017. 